\title{
Public perceptions of endometriosis: perspectives from both genders
}

\author{
DIVYA K. SHAH ${ }^{1}$, MOLLY B. MORAVEK ${ }^{2}$, ANJEL VAHRATIAN $^{2}$, VANESSA K. DALTON ${ }^{2} \&$ \\ DAN I. LEBOVIC ${ }^{3}$
}

${ }^{1}$ Department of Obstetrics and Gynecology, Division of Reproductive Endocrinology and Infertility, Brigham and Women's Hospital, Boston, Massachusetts, USA, ${ }^{2}$ Department of Obstetrics and Gynecology, University of Michigan, Ann Arbor, Michigan, USA, and ${ }^{3}$ Department of Obstetrics and Gynecology, Division of Reproductive Endocrinology and Infertility, University of Wisconsin-Madison, Madison, Wisconsin, USA

\begin{abstract}
Objective. Many women with endometriosis experience significant delay between the onset of symptoms and definitive diagnosis. Much is published on physician awareness of endometriosis and on the experiences of women with the condition. There is a paucity of data, however, surrounding perceptions of endometriosis in the general population. This study aims to assess knowledge of endometriosis among individuals of both genders. Design. Survey study. Setting. Family waiting room of a large university hospital. Population. A total of 543 men and women. Methods. Surveys were distributed to men and women over the age of 18 in the family waiting room of a large university hospital. Main outcome measures. A series of questions regarding the etiology, symptoms, and treatments for endometriosis were combined into a composite knowledge score. Results. Knowledge of endometriosis was positively correlated with female gender, education level, regular health care, and exposure to individuals with the disease. Women diagnosed with endometriosis were more likely to have discussed symptoms of the condition with their physicians than women without the diagnosis. Conclusions. Individuals of both genders have limited knowledge of the signs and symptoms of endometriosis, which may contribute to the delay in diagnosis of the condition.
\end{abstract}

Key words: Endometriosis, education, public awareness

\section{Introduction}

While the true prevalence of endometriosis is difficult to quantify, it is estimated that the disease affects approximately 1 in 10 reproductive aged women in the United States (1). In 2000, endometriosis accounted for over 86,000 hospital admissions, with estimated mean charges amounting to over $\$ 884$ million in hospitalization costs alone (2).

Despite the significant impact of endometriosis on both individual patients as well as the health care system, epidemiologic studies reveal concerning trends regarding the current state of diagnosis and access to treatment. Studies in the United States and other industrialized nations have found that a majority of women with endometriosis have been serially evaluated by multiple providers, have had their symptoms dismissed or trivialized by health care professionals, and ultimately experience a significant delay between the onset of symptoms of endometriosis and its definitive diagnosis (3). Data collected from over 4,000 women as part of the Endometriosis Association's 1998 North American Member Survey (4) reveal that $47 \%$ of women reported having seen a health care provider five or more times before being diagnosed or referred. It was seen that 61 percent of women were told 'nothing was wrong' when they first presented to a physician. The delay between onset of symptoms and actual diagnosis of disease averaged 9.28 years: the average woman took 4.67 years to report her symptoms to a physician, and the average physician took another 4.61 years to diagnose the disease.

Correspondence: Divya Shah, Center for Infertility and Reproductive Surgery, Department of Obstetrics and Gynecology, Brigham and Women's Hospital, 75 Francis Street, Boston, MA 02115, USA. E-mail: dkshah@partners.org 
There exist large bodies of literature regarding physician awareness of endometriosis as well as evaluating the experiences of women already diagnosed with the disease (1). There is a paucity of data, however, surrounding the perceptions of the general population with respect to endometriosis as a disease, its symptoms, and its role as a potential cause of pelvic pain. This study aims to explore the knowledge and attitudes of individuals of both genders. By serving as a baseline assessment of the general public's knowledge of endometriosis, these data may guide health education efforts to promote awareness of the condition and decrease the delay in its diagnosis.

\section{Methods}

Surveys were distributed to a convenience sample of men and women present in a university hospital surgery family waiting room over a seven-month period between November 2006 and June 2007. Specific permission was granted from the administrative offices of the main hospital to conduct subject recruitment in this location. The study was approved for exempt status (exemption \#2 of 45 CFR 46.101.b) by the University of Michigan Medical School Institutional Review Board. Any English-speaking individual over the age of 18 was eligible to participate. Our research assistant administered a brief recruitment script that described the purpose of the research, its voluntary nature, and information on risks and benefits. Participants were asked to read and initial this script, indicating their consent. No personal identifying information was collected. Of the 543 individuals who were approached regarding the project, 418 consented to participate and provided complete demographic information, for an overall response rate of $77 \%$. The response rate was $71.5 \%$ among males (143 of 200 approached) and $80.2 \%$ among females (275 of 343 approached).

Following a thorough literature review of similar work, the authors drew on their collective clinical and epidemiologic experience in the field of endometriosis to develop the survey instrument. The survey was pretested in a pilot group of individuals for clarity, comprehensibility, and length prior to its formal administration. Demographic information, including a participant's age, gender, education level, ethnic background, source of employment, and access to annual health care were assessed. A participant's personal experience with endometriosis was assessed based on knowledge of an individual with the disease, and for female respondents, by self-reported diagnosis of endometriosis.
To measure a participant's knowledge about endometriosis, the authors developed a series of four multiple-choice items regarding the symptoms, etiology, and treatment of the condition that were combined into a composite score (Table 1). The composite score was weighted to preferentially reflect an individual's accuracy in identifying the presenting symptoms of endometriosis as follows: 2 points if participants could identify endometriosis as one of nine potential sources of lower abdominal or pelvic pain (maximum 2 points), 1 for identifying the uterus as the body part from which the disease originates (maximum 1 point), 2 for correctly identifying symptoms of endometriosis (participants could select from 9 responses, with four correct answers, for a maximum 8 points), and 1 for correctly identifying potential treatments for the disease (participants could select from twelve responses, with five correct answers, for a maximum 5 points), for a total score of 16 points. Points were not deducted for incorrect answers. For purposes of descriptive analysis, knowledge scores were clustered into low (less than 5), average (5-8), and high (9-16) ranges. The decision was made to evaluate the composite score as a categorical rather than a continuous variable in order to facilitate clear visual comparisons between the various demographic groups.

All analyses were performed using Statistical Analysis Software (SAS) Version 9.1 for Windows (SAS Institute Inc., Cary, North Carolina, USA). Descriptive analyses were conducted to ascertain demographic characteristics of the study population and to document variations in health knowledge by sex,

Table 1. Questions comprising composite gynecologic knowledge score.

1. If a woman complained to you of lower abdominal or pelvic pain, you might suggest the pain could be caused by which of the following? ( 2 points for including endometriosis)

2. Which body part does endometriosis come from? (1 point for identifying uterus)

3. Which of the following are symptoms of endometriosis? (2 points for each correctly identified symptom including: irregular vaginal bleeding, heavy vaginal bleeding, pain with sexual intercourse, painful periods)

4. Which of the following are ways to treat endometriosis? [1 point for each correctly identified treatment including: anti-estrogen hormones, birth control pills, progesterone, hysterectomy (taking out the uterus), laparoscopic surgery to remove endometriosis]

The composite score was weighted as follows: 2 points if participants could identify endometriosis as a source of pelvic pain (maximum 2 points), 1 for identifying the uterus as the body part from which the disease originates (maximum 1 point), 2 for correctly identifying symptoms of endometriosis (maximum 8 points), and 1 for correctly identifying potential treatments for the disease (maximum 5 points), for a total score of 16 points. Points were not deducted for incorrect answers. 
personal history of endometriosis, and other demographic characteristics where appropriate. For continuous variables, mean and standard deviations were calculated. For categorical data, percentages were calculated and the chi-squared test was used to assess statistical significance where relevant.

\section{Results}

Survey participants included 275 women $(66 \%)$ and $143(34 \%)$ men (Table 2). The mean age of participants was 50.2 years $(S D=14.2)$. Of the 275 female respondents, $181(65 \%)$ reported a personal history of dysmenorrhea. A total of $44(16 \%)$ reported a personal history of endometriosis. A majority of these women ( 40 out of 44 ) were under the age of 60 . The racial distribution of the participants was reflective of the demographics of the county, with a majority of those surveyed self-identifying as Caucasian (88\%). A majority of participants reported a level of education beyond a high school degree, and approximately $15 \%$ of survey participants were employed in the health professions. Female respondents were more likely to seek annual health care than their male counterparts (87 vs. $71 \%, p<0.05$ ).

Knowledge of endometriosis was uniformly lower among men as compared to women. Of female respondents $91 \%$ had heard of a condition called endometriosis, and $69 \%$ were able to identify the uterus as the organ from which it originates. By comparison, $57 \%$ of male respondents had heard of the condition, and only $39 \%$ were able to identify its

Table 2. Demographic characteristics of the study population.

\begin{tabular}{lccc}
\hline & $\begin{array}{c}\text { Female (\%) } \\
n=275\end{array}$ & $\begin{array}{c}\text { Male (\%) } \\
n=143\end{array}$ & $\begin{array}{c}\text { Overall (\%) } \\
n=418\end{array}$ \\
\hline Age & & & \\
$\quad<19$ years & 1 & 0 & 1 \\
19-35 years & 17 & 14 & 22 \\
36-45 years & 22 & 22 & 39 \\
46-60 years & 39 & 38 & 23 \\
$>$ 60 years & 22 & 26 & \\
Race & & & 88 \\
$\quad$ White & 87 & 88 & 12 \\
$\quad$ Non-white & 13 & 12 & 5 \\
Education & & 6 & 75 \\
$\quad<$ High school & 6 & 15 & \\
$\quad$ High school & 22 & 80 & 15 \\
$\quad>$ High school & 72 & & 85 \\
Employment in health \\
profession
\end{tabular}

Demographic variables including age, gender, race, employment, and education level are self-reported by study participants. organ of origin. When asked to select among potential etiologies for pelvic pain, pre-menstrual syndrome was the top response among male respondents $(39 \%)$, while women were most likely to choose endometriosis (67\%). Of male respondents, $57 \%$ (vs. $22 \%$ of female respondents) reported having no idea what the symptoms of endometriosis were, and another $55 \%$ (vs. $26 \%$ of females) could not identify a single treatment option for the disease.

Endometriosis composite knowledge scores were stratified by the various demographic factors assessed in the survey. Composite knowledge scores were found to be independent of respondent age (data not shown), but positively correlated with education level, employment in the health professions, selfreported access to annual health care, and self-reported exposure to individuals with the disease (Figure 1). Composite knowledge scores also displayed a strong correlation with gender, with a majority of male respondents having a 'low' knowledge score.

When the sample was restricted to female respondents, composite knowledge scores were again independent of age (data not shown) but were significantly higher among women who were themselves diagnosed with endometriosis as compared to both the general population of female respondents, as well as to women who merely reported knowing someone with the disease (Figure 1). Women with a diagnosis of endometriosis were significantly more likely to be able to recognize the disease as a potential source of pelvic pain, as well as accurately identify its etiology, symptoms, and potential treatment options.

Among women who reported a personal history of dysmenorrhea, composite knowledge scores were notably higher in those that chose to discuss the symptom with their physicians. Women with a diagnosis of endometriosis were significantly more likely to have discussed symptoms of the condition with their physicians than women without the diagnosis (95 vs. $69 \%$, respectively, $p=0.001$ ).

\section{Discussion}

This study demonstrates that a significant proportion of the general public has limited or no knowledge about endometriosis. We identified a significant gender disparity in the public awareness of the typical presentation of endometriosis, which may contribute to the social isolation experienced by many women with the condition. Additionally, the limited knowledge of endometriosis as a potential cause of pelvic pain may contribute to the delay in diagnosis noted by many other groups (4-7). 


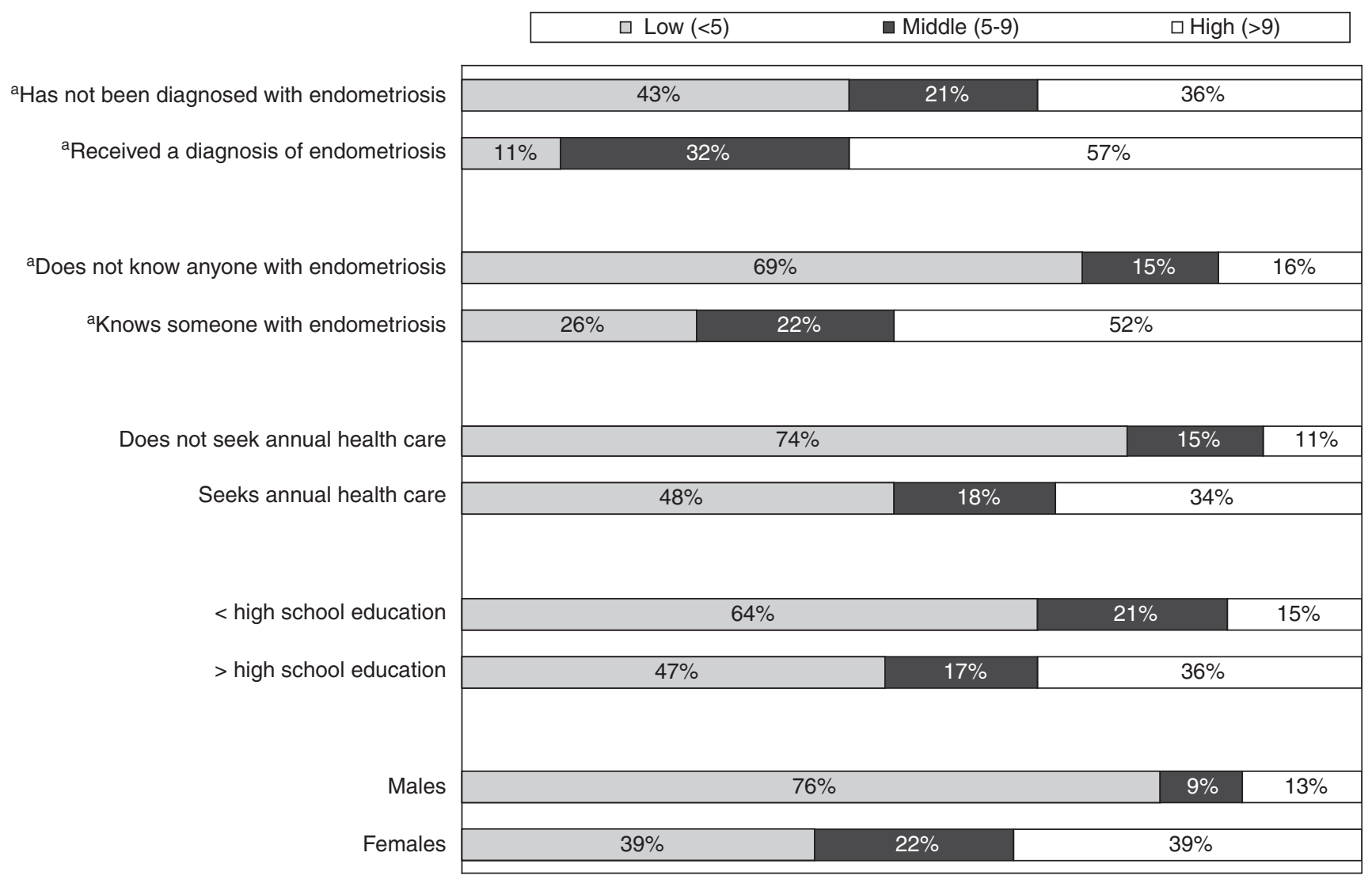

Figure 1. Knowledge scores stratified by demographic variables and personal experience with endometriosis ${ }^{\mathrm{a}}$. Composite knowledge scores are clustered into low $(<5)$, average (5-8), and high (9-16) ranges. Numerical values represent the percentage of individuals achieving a score within the specified range. Scores were positively correlated with female gender, education level, employment in the health professions, access to annual health care, and self-reported exposure to individuals with endometriosis. $p$ Values are $<0.001$ for all comparisons shown.

${ }^{\text {a }}$ Sample restricted to female participants.

A study by Lemaire (2) reported a high degree of emotional distress among women with endometriosis who felt they lacked information about the disease. In our study population, knowledge of endometriosis was positively correlated with annual contact with a health care provider, education level, employment in the health professions, and a respondent's exposure to individuals with the condition. Knowledge scores were highest among women who reported a personal diagnosis of endometriosis or women who reported knowing someone with the condition.

Focus groups of women with endometriosis have revealed a strong desire for 'the production and dissemination of information not only to women with endometriosis, but also to teachers, employers, partners, and family members' (3). This sentiment may represent a response to the perception of being 'misunderstood' by family, friends, and colleagues. For example, $39 \%$ of males in our study identified 'premenstrual syndrome' as the most likely cause of pelvic pain in women. Additionally, knowledge of endometriosis in our sample was consistently lower in individuals of both genders who did not personally know anyone affected with the disease. The presence of a close friend or family member who 'took their complaints seriously' was identified by a recent study as the most common catalyst that led women to seek medical treatment for endometriosis (8), suggesting that increased public awareness of endometriosis may help facilitate earlier medical intervention for women with the disease.

Prior studies on public awareness of conditions such hypertension and colorectal cancer have suggested that utilization of available screening tests may be improved through increased community education $(9,10)$. Additionally, recent studies in type 2 diabetics have consistently demonstrated short-term improvement in clinical outcome in response to educational programming in specific ethnic subpopulations $(11,12)$. It is hoped that by providing a baseline assessment of public knowledge of endometriosis, this study may similarly guide targeted health education to facilitate its timely diagnosis. 
Several limitations should be considered when interpreting our results. First, our findings may not be generalizable to the larger U.S. population, as the use of a surgery family waiting room as our recruitment location biased our demographics toward an older, more educated population. Although the ethnic makeup of our study population was representative of that of the county, the older average age of our study participants resulted in an undersampling of younger women and their partners. Despite the older average age of study participants, however, $91 \%$ of female participants who reported a personal history of endometriosis were under the age of 60 . This may reflect increased diagnosis of endometriosis in recent years or may represent a recall bias among postmenopausal women. Second, while $65 \%$ of female respondents reported a personal history of dysmenorrhea, our survey did not assess the severity of symptoms. It is likely that women with relatively mild symptoms may not choose to discuss them with their physicians. Finally, our study design is unable to assess causality between a participant's knowledge of endometriosis and the time to diagnosis of the condition. For example, we cannot distinguish between the influence that pre-existing knowledge of endometriosis has on diagnosis of the condition, and the increased knowledge that results from an individual having already received this diagnosis. While we can postulate that increased public knowledge of endometriosis will facilitate timely diagnosis of the condition as has been demonstrated in other disease models $(9,10)$, this conclusion is not directly demonstrated by the data.

Several groups have documented the significant delay between the initial symptoms of endometriosis and a woman's first contact with a physician (4-7). This delay is partially attributed to dismissal or normalization of pelvic pain symptoms by spouses, family, and employers $(3,8,13)$. Early diagnosis and treatment of endometriosis may reduce emotional suffering as well as long-term complications such as pain and infertility. Increasing public awareness of endometriosis may help facilitate early diagnosis and alleviate the social isolation reported by many women with the condition.
Declaration of interest: The authors report no conflicts of interest. The authors alone are responsible for the content and writing of the paper.

\section{References}

1. Prentice A. Clinical review: endometriosis. BMJ. 2003; 323:93-96.

2. Lemaire GS. More than just menstrual cramps: symptoms and uncertainty among women with endometriosis. J Obst Gynecol Neonatal Nurs. 2004;33:71-79.

3. Cox H, Henderson L, Andersen N, Cagliarini G, Ski C. Focus group study of endometriosis: struggle, loss and the medical merry-go-round. Int J Nurs Pract. 2003;9:2-9.

4. Ballweg ML. Impact of endometriosis on women's health: comparative historical data show that the earlier the onset, the more severe the disease. Best Pract Res Clin Obstet Gynaecol. 2004; 18:201-18

5. Hadfield R, Mardon H, Barlow D, Kennedy S. Delay in the diagnosis of endometriosis: a survey of women from the USA and the UK. Hum Reprod. 1996;114:878-80.

6. Husby GK, Haugen RS, Moen MH. Diagnostic delay in women with pain and endometriosis. Acta Obstet Gynecol Scand. 2003;827:649-53.

7. Dmowski WP, Lesniewicz R, Rana N, Pepping P, Noursalehi $M$. Changing trends in the diagnosis of endometriosis: a comparative study of women with pelvic endometriosis presenting with chronic pelvic pain or infertility. Fertil Steril. 1997;672:238-43.

8. Manderson L, Warren N, Markovic M. Circuit Breaking: pathways of treatment seeking for women with endometriosis in Australia. Qual Health Res. 2008;16:522-34.

9. Wyatt SB, Akylbekova EL, Wofford MR, Coady SA, Walker ER, Andrew ME, et al. Prevalence, awareness, treatment and control of hypertension in the Jackson Heart Study. Hypertension. 2008;51:650-6.

10. Ling BS, Klein WM, Dang Q. Relationship of communication and information measures to colorectal cancer screening utilization: results from HINTS. J Health Commun. 2006;11:181-90.

11. Metghalchi S, Rivera M, Beeson L, Firek A, De Leon M, Cordero-Macintyre ZR, et al. Improved clinical outcomes using a culturally sensitive diabetes education program in a Hispanic population. Diabetes Educ. 2008;34:698-706.

12. Hawthorne, K, Robles Y, Cannings-John R, Edwards AG. Culturally appropriate health education for type 2 diabetes mellitus in ethnic minority groups. Cochrane Database Syst Rev. 2008;3:CD006424.

13. Denny E. Women's experience of endometriosis. J Adv Nurs. 2004;46:641-48. 